\title{
Investigation of Antibiotic Release from Bone Allograft in an Experiment on Rabbits
}

\author{
Berik Tuleubayev ${ }^{1}$ (D), Alexandr Rudenko ${ }^{2 *}$ (D) \\ ${ }^{1}$ Department of Surgical Diseases, Non-commercial Corporation "Karaganda Medical University", "Multifunctional Regional \\ Center of Traumatology and Orthopedics named after Professor H.Zh.Makazhanov", Karaganda, Kazakhstan; 'Department \\ of Emergency Trauma, Multifunctional, "Regional Center of Traumatology and Orthopedics named after Professor H.Zh. \\ Makazhanov", Karaganda, Kazakhstan
}

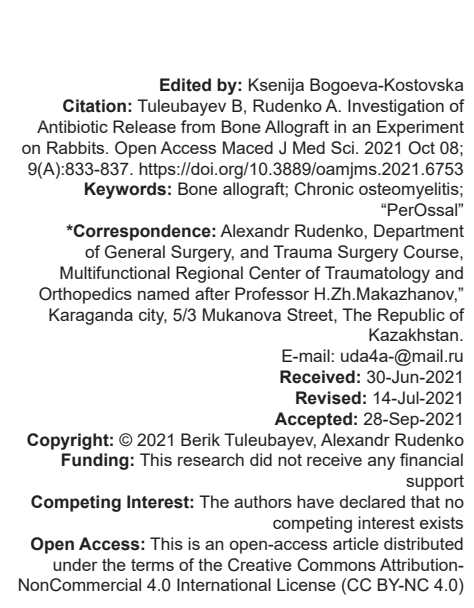

\section{Abstract}

BACKGROUND: The treatment of chronic osteomyelitis, despite the use of new methods, is still an urgent problem. Local use of antibacterial drugs in combination with systemic antibiotic therapy has become popular in recent decades. Autologous bone grafts are considered ideal for bone defects filling. Different methods of allograft preparation may have differences in the rate and duration of antibiotic release. Moreover, it can affect the effectiveness of microbia agent eradication. The study analyzed the differences in the release of gentamicin from different types of allografts in dynamics and methods of preparation: «PerOssal» medium, whole bone allograft soaked in antibiotic, whole bone allograft, welded with an antibiotic, and perforated bone allograft soaked in an antibiotic solution.

AIM: The objective of the study was to study the stability of antibiotic release and to determine the effectiveness of local transport systems. Evaluation of the difference in gentamicin release from different types of allografts in dynamics and methods of preparation had been realized: "PerOssal" medium, whole bone allograft soaked in antibiotic, whole bone allograft welded with an antibiotic, and perforated bone allograft, soaked in antibiotic solution.

MATERIALS AND METHODS: The research was conducted between September 2020 and March 2021. The experiments were performed on 120 laboratory rabbits (weight $-3000-3500 \mathrm{~g}$, age $-6-8$ months), which were divided into four groups (30 animals in each group). Group 1 consisted of animals treated with "PerOssal." The whole bone allograft soaked in an antibiotic was used in the treatment of animals of Group 2. The whole bone allograft, welded with an antibiotic, was used in the treatment of animals of Group 3. Perforated bone allograft soaked in an antibiotic was used in Group 4. Osteomyelitis of the proximal femur was formed in experimental animals.

RESULTS AND DISCUSSION: Statistically insignificant decrease in the concentration of gentamicin was observed by the $7^{\text {th }}$ day in all experimental groups. In rabbits whose bone defect was filled with a whole bone allograft welded with antibiotic and perforated bone allograft impregnated with an antibiotic (Groups 3 and 4), the most stable concentration of gentamicin was noted throughout the study period. Statistically significant differences were revealed between the experimental groups in relation to the dynamics of changes in the concentration of gentamicin in blood plasma. It was found that the group using the biodegradable material "PerOssal" on the $1^{\text {st }}$ day showed a high concentration of the antibiotic in the blood plasma. However, by the $2^{\text {nd }}$ day, a lower concentration of the antibiotic was recorded compared to all comparison groups of the bone allograft.

CONCLUSIONS: The results of the analysis of the dynamics of gentamicin concentration may indicate significant differences between the methods of graft preparation, especially in the relationship with antibiotic release into the blood plasma. The most stable antibiotic concentration was registered in the groups of animals that underwent the filling of bone defect using a whole bone allograft welded with an antibiotic and a perforated bone allograft impregnated with antibiotic. A significant decrease of gentamicin concentration in the femur homogenate by the $7^{\text {th }}$ day after transplantation was observed when using a whole bone allograft impregnated with an antibiotic. At the same time, a stable concentration of the antibiotic in the blood plasma was registered. The highest initial antibiotic concentration in the homogenate with a gradual decrease over 7 days was observed when using the antibioticimpregnated biodegradable material "PerOssal."

\section{Introduction}

The treatment of chronic osteomyelitis, despite the use of new methods, is still an urgent problem. Surgical debridement of the infection nidus and adequate antibiotic therapy is currently the main components of treatment [1]. Local use of antibacterial drugs in combination with systemic antibiotic therapy has become popular in recent decades [2]. Local use of antibiotics is devoid of such disadvantages inherent in systemic administration as a high incidence of adverse drug reactions, a short half-life, and a low concentration at the infection nidus. The approaches to the local delivery of antibiotics are constantly being improved to increase the efficiency [3], [4]. Studies have shown the 
effectiveness of antibiotics delivery in the composition of bone allografts, such as calcium sulfate, bone cement, polymethyl methacrylate (PMMA), autologous bone, and allograft. An important characteristic of this approach is the possibility of bone cavity filling after surgical debridement with a material that contributes not only to the elimination of the pathogen but also to the growth of bone tissue into the allograft cavity while preserving the primary bone structure.

Those materials as PMMA are the most popular among surgeons. Their use has become the "gold standard" in the surgical treatment of chronic osteomyelitis. This antibiotic delivery system can guarantee the advantage of local release of high concentrations at the infection nidus and simultaneously providing the dead space that occurs during surgical debridement [5]. However, PMMA has some drawbacks that require the further research and development. One of these disadvantages is the fact that PMMA can serve as a substrate for bacterial colonization when the release of the loaded antibiotic is reduced [6]. In addition, PMMA is not biodegradable and requires the repeated surgery. Bone auto- and allografts, as well as calcium sulfate, are devoid of this deficiency. Antibiotic-loaded calcium sulfate particles had comparable efficacy in the treatment of chronic osteomyelitis compared to antibiotic-loaded PMMA [7]. Moreover, calcium sulfate is capable of antibiotic releasing at a relatively constant rate. This helps to maintain the therapeutic antibiotic concentration in the wound for a longer time. It is worth noting that antibiotic delivery devices made from calcium sulfate also have drawbacks [8]. Destabilization of the structure during degradation is one drawback. A defect in bone tissue before the stabilization of the fragments can be formed in the case of early lysis of this type of allograft. However, mechanical stabilization of the bone structure is of great importance in the treatment of chronic osteomyelitis [9].

The reconstruction of bone defects caused by tumors, infections, and trauma is a complex area of orthopedic medicine [1], [2]. Various restoring methods of lost bone tissue are currently used [2], [3]. The use of autogenous or allogeneic bone grafts is one such method. More than 2 million bone transplants are performed worldwide every year. This is the second most common tissue transplantation after blood transfusion [2], [4], [5]. Grafts perform the functions of mechanical support and osteoregeneration, including osteoconduction, osteoinduction, and osteogenesis [6]. The clinical choice of biomaterials depends on many factors, such as etiopathogenetic aspects, severity of damage, financial and economic costs, and drew [1], [7]. The study of the biological properties of various materials is used for bone grafting, and the search for new materials is an urgent task [2], [4].

Autologous bone grafts contain bone matrix, growth factors, osteoblasts [10], [11], and therefore are popular. The disadvantages of using autologous grafts are the limited amount of material and complications at the place of material collection.

The market of orthopedic bone substitutes for bone tissue is increasing due to the growing demand for bone grafts [12].

"PerOssal" is a synthetic osteoconductive resorbable bone graft substitute, a composite of nanocrystalline hydroxyapatite and calcium sulfate. Nanocrystalline hydroxyapatite creates a specific surface that promotes protein adhesion, provides growth factors, and promotes osteostimulation of the surrounding tissues. The network of nano- and micropores of the drug makes it possible to fill with antibiotics and ensures their controlled long-term release.

The combination of an allograft with an antibiotic is also often used to treat or prevent bone infection [8], [9]. There is some research on the effect of antibiotics on bone regeneration. The use of antibiotics for bone grafts is controversial [10], [11]. Fassbender et al. reported that the use of lyophilized bone allografts in combination with topical gentamicin does not interfere with bone regeneration [12]. This is consistent with an earlier report that indicated successful treatment of defects using the same treatment scheme [13]. Durmuşlar et al. also reported better results when combining antibiotics with bone grafts than when using graft alone. In contrast, several other studies have shown that the integration of antibiotics into the graft material has a depressive effect on bone formation. However, all of these studies used lyophilized bone autografts. There are no data on the antibiotic impregnation of heat-treated bone grafts in the available literature.

Different methods of allografts preparation can be characterized by significant differences in the rate and duration of antibiotic release, which, in turn, affects the eradication efficiency of the microbial agent in the focus of infection.

Previously, it have been conducted the studies on the stability of antibiotic release from the bone allograft for 6 weeks [13]. The purpose of this research was to study the stability of antibiotic release and to determine the effectiveness of local transport systems. Evaluation of the difference in gentamicin release from different types of allografts in dynamics and methods of preparation had been realized: "PerOssal" medium, whole bone allograft soaked in antibiotic, whole bone allograft welded with an antibiotic, and perforated bone allograft, soaked in antibiotic solution.

\section{Materials and Methods}

The research was conducted between September 2020 and March 2021. 
The experiments were performed on 120 laboratory rabbits (weight - 3000 - $3500 \mathrm{~g}$, age - 6-8 months), which were divided into four groups (30 animals in each group). Group 1 consisted of animals treated with "PerOssal." The whole bone allograft soaked in an antibiotic was used in the treatment of animals of Group 2. The whole bone allograft, welded with an antibiotic, was used in the treatment of animals of Group 3. Perforated bone allograft soaked in an antibiotic was used in Group 4. The study protocol was approved by the Bioethics Committee of the Non-Profit Joint Stock Company "Karaganda medical university."

\section{Osteomyelitis of the proximal femur was formed in experimental animals}

Staphylococcus aureus was injected into the upper third of the femur of laboratory animals to simulate osteomyelitis. A skin incision in the projection of the proximal femur was made to simulate osteomyelitis, the proximal femoral epiphysis was isolated using a drill of the optimal size, and then osteoperforation of the femur was performed. Infection of the femoral epiphysis was carried out with a cotton ball with $S$. aureus culture strain. The animals were included in the further phase of the trial in 14 days after the intervention, if osteomyelitis was confirmed clinically and radiologically. Bone defects in animals of Group 1 were filled with biodegradable material "PerOssal" impregnated with an antibiotic. Bone defects of animals of Group 2 were filled with whole bone allograft impregnated with antibiotic. The whole bone allograft was impregnated with an antibiotic after heat treatment in "LOBATOR SD-2" apparatus. Bone defects of animals of Group 3 were filled with a whole bone allograft, welded with an antibiotic during heat treatment in "LOBATOR SD-2" apparatus. Bone defects in Group 4 rabbits were filled with a perforated bone allograft impregnated with an antibiotic. The perforated bone allograft was impregnated with an antibiotic after heat treatment in "LOBATOR SD-2" apparatus.

The rabbits were led into inhalation anesthesia using a special anesthesia chamber for animals and anesthetic sevoflurane to obtain a tissue homogenate around the osteomyelitis focus. After pre-treatment with a solution of povidone-iodine in the region of the distal femur of rabbits, a skin incision up to $3 \mathrm{~cm}$ was made, and the femur was isolated in layers. Tissues around the osteomyelitis focus were removed with a scalpel and scissors. In parallel, blood was taken from the vessels of the rabbit's ear to study the concentration of gentamicin in the blood plasma.

Data on gentamicin release from the bone allograft were recorded by measuring its concentration in 1,3 , and $6 \mathrm{~h}$ and $1,2,3,4,5,6$, and 7 days after transplantation using high-performance liquid chromatography. An Agilent 1260 Infinity chromatographic system (Agilent Technologies, USA) was used to analyze the antibiotic concentration in the test samples according to the manufacturer's instructions.

Statistical analysis and data visualization were carried out using the $\mathrm{R}$ 4.1.0 statistical computing environment ( $R$ Foundation for Statistical Computing, Vienna, Austria) and the additional rms 6.2-0 package. Descriptive statistic was presented as the median (1, 3 quartiles). Proportional odds models for quantitative variables with the inclusion of the term interactions between these variables were used to compare homogenate and plasma gentamicin concentrations between groups of animals and time periods after intervention. Pairwise comparisons between groups were performed using Tukey's contrasts implemented in the emmeans 1.6.1 package. Differences were considered statistically significant at $p<0.05$.

\section{Results and Discussion}

The results of evaluation of the dynamics of gentamicin concentration in the femur homogenate are presented in Figure 1.

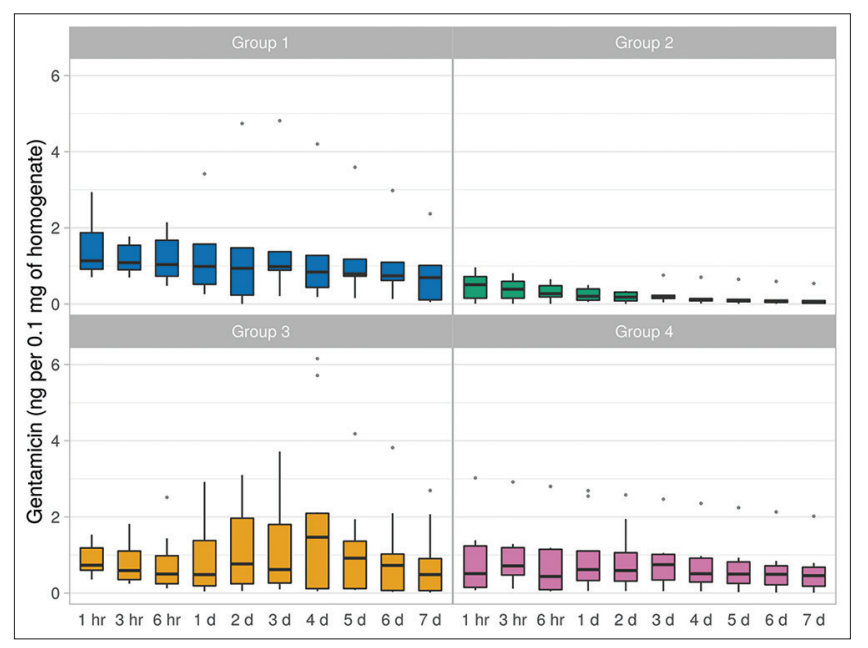

Figure 1: Dynamics of gentamicin concentration in the homogenate of the femur in the experimental groups of animals that underwent the bone defect filling: Group 1 - "PerOssal;" Group 2 - whole bone allograft impregnated with an antibiotic; Group 3 - whole bone allograft, welded with an antibiotic; Group 4 - perforated bone allograft impregnated with an antibiotic

Statistically insignificant decrease in the concentration of gentamicin was observed in all experimental groups by day 7. There was a gradual decrease of gentamicin concentration in the group of animals using the biodegradable material "PerOssal" impregnated with an antibiotic (Group 1) - from $1.13(0.92-1.87) \mathrm{ng} / \mathrm{mg}$ in $1 \mathrm{~h}$ to $0.69(0.11-1.01) \mathrm{ng} / \mathrm{mg}$ in 7 days after transplantation $(p=0.9)$. The most significant decrease of gentamicin concentration after transplantation was noted in animals 
whose bone defect was filled with a whole bone allograft impregnated with gentamicin (Group 2)-from 0.51 (0.15$0.72) \mathrm{ng} / \mathrm{mg}$ in $1 \mathrm{~h}$ to 0.05 (0.02-0.09) $\mathrm{ng} / \mathrm{mg}$ in 7 days after transplantation. However, these differences were not statistically significant $(p=0.5)$.

The most stable gentamicin concentration throughout the study period was observed in rabbits, whose bone defect was filled with a whole bone allograft welded with an antibiotic and a perforated bone allograft impregnated with an antibiotic (Groups 3 and 4) - from $0.73(0.60-1.18) \mathrm{ng} / \mathrm{mg}$ and $0.51(0.15-1.24) \mathrm{ng} / \mathrm{mg}$ after $1 \mathrm{~h}$ to $0.49(0.06-0.91) \mathrm{ng} /$ mgand $0.46(0.18-0.68) \mathrm{ng} / \mathrm{mg}$ after 7 days, respectively $(p=0.9)$. However, gentamicin concentration was slightly higher in animals with whole bone allograft welded with an antibiotic (Group 3), than in the group of rabbits whose bone defect was filled with a perforated bone allograft impregnated with an antibiotic (Group 4).

Statistically significant differences between the experimental groups were found in relation to the dynamics of gentamicin concentration changes in blood plasma $(p<0.0001)$. Thus, the expressed non-linear dynamics of concentration was observed in the group of animals whose bone defect was filled with biodegradable material "PerOssal" impregnated with an antibiotic (Group 1): On the $1^{\text {st }}$ day, an increase in concentration was observed from 34.21 (30.24-47.12) $\mathrm{ng} / \mathrm{ml}$ to $73.71(72.06-82.01) \mathrm{ng} / \mathrm{ml}$.

Subsequently, a further statistically significant decrease in concentration was registered - to $5.02 \mathrm{ng} / \mathrm{ml}$ on the 7th day of the experiment $(p<0.0001)$. The less pronounced changes in the concentration of gentamicin were observed during the observation period in other groups. A gradual decrease in concentration was noted in animals of Group 2 - from $39.59 \mathrm{ng} / \mathrm{ml}$ to 14.66 $\mathrm{ng} / \mathrm{ml}$ on the 4 th day after transplantation $(\mathrm{p}=0.4)$. A statistically insignificant $(p=0.3)$ increase of gentamicin

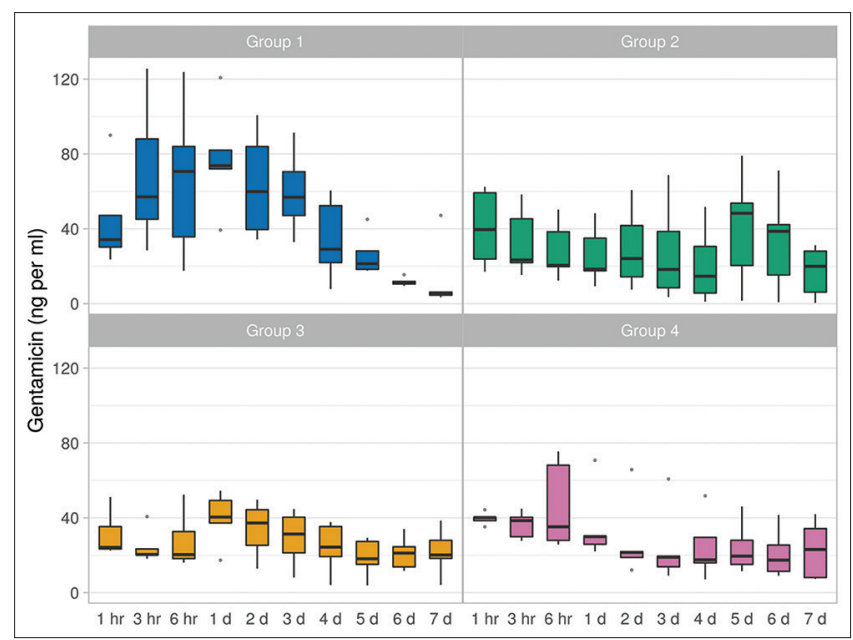

Figure 2: Dynamics of gentamicin concentration in blood plasma of animals that underwent the bone defect filling: Group 1 "PerOssal;" Group 2 - whole bone allograft impregnated with an antibiotic; Group 3 - whole bone allograft, welded with an antibiotic, Group 4 - perforated bone allograft impregnated with an antibiotic concentration was observed on the 5th day - to 48.31 $\mathrm{ng} / \mathrm{ml}$. Subsequently, a decrease was recorded by the 7th day after transplantation - to $19.97 \mathrm{ng} / \mathrm{ml}(\mathrm{p}=$ 0.4 ), the most stable gentamicin concentration in blood plasma was observed in Group 3: $24.20 \mathrm{ng} / \mathrm{ml}$ after $1 \mathrm{~h}$ and $20.14 \mathrm{ng} / \mathrm{ml}$ after 7 days $(p=0.8)$. A gradual decrease of antibiotic concentration was noted in the group of animals whose bone defect was filled with a perforated bone allograft welded with an antibiotic (Group 4) - from $40.13 \mathrm{ng} / \mathrm{ml}$ after $1 \mathrm{~h}$ to $23.10 \mathrm{ng} / \mathrm{ml}$ after 7 days $(p=0.8)$ (Figure 2).

\section{Conclusions}

The results of the analysis of gentamicin concentration dynamics may indicate significant differences between the methods of graft preparation, especially with respect to antibiotic release into blood plasma. The most stable antibiotic concentration was observed in the groups of animals that underwent filling of the bone defect using a whole bone allograft welded with an antibiotic (Group 3) and a perforated bone allograft impregnated with an antibiotic (Group 4). A significant decrease of gentamicin concentration in the homogenate of the femur was observed when using a whole bone allograft impregnated with an antibiotic (Group 2) by the $7^{\text {th }}$ day after transplantation. Moreover, this group was characterized by a stable antibiotic concentration in blood plasma. It is also worth noting that the lowest initial antibiotic concentration in the homogenate was noted when using this type of bone material, which makes it possible to make an assumption about the lowest absorption rates.

The highest initial antibiotic concentration in the homogenate with a gradual decrease over 7 days was observed when using the biodegradable material "PerOssal" impregnated with an antibiotic (Group 1). However, in the blood plasma, significant fluctuations in concentration were noted. The mechanisms of such changes in concentration at this stage cannot be explained and require the further study. However, the indicators of the presented type of allograft preparation may indicate the possibility of antibiotic resistance development of pathogenic microflora due to sharp drops and early antibiotic release and, as a consequence, the likely low clinical efficacy.

\section{References}

1. Anagnostakos K, Hitzler P, Pape D, Kohn D, Kelm J. Persistence of bacterial growth on antibiotic-loaded beads: Is it actually a problem? Acta Orthop. 2008;79(2):302-7. https://doi. org/10.1080/17453670710015120 
PMid:18484259

2. Luo S, Jiang T, Yang $Y$, Yang $X$, Zhao J. Combination therapy with vancomycin-loaded calcium sulfate and vancomycinloaded PMMA in the treatment of chronic osteomyelitis. BMC Musculoskelet Disord. 2016;17(1):502. https://doi.org/10.1186/ s12891-016-1352-9

PMid:28007027

3. El-Husseiny M, Patel S, MacFarlane RJ, Haddad FS. Biodegradable antibiotic delivery systems. J Bone Joint Surg. 2011;93(2):151-7.

PMid:21282751

4. Gauland C. Managing lower-extremity osteomyelitis locally with surgical debridement and synthetic calcium sulfate antibiotic tablets. Adv Skin Wound Care. 2011;24(11):515-23. https://doi. org/10.1097/01.asw.0000407647.12832.6c

PMid:22015750

5. Heijink A, Yaszemski MJ, Patel R, Rouse MS, Lewallen DG, Hanssen AD. Local antibiotic delivery with OsteoSet, DBX, and collagraft. Clin Orthop Relat Res. 2006;451:29-33. https://doi. org/10.1097/01.blo.0000229319.45416.81

PMid:16906070

6. Henry SL, Galloway KP. Local antibacterial therapy for the management of orthopaedic infections. Pharmacokinetic considerations. Clin Pharmacokinet. 1995;29(1):36-45. https:// doi.org/10.2165/00003088-199529010-00005

PMid:7586897

7. Vaishya R, Pokhrel A, Agarwal AK, Vijay V. Current status of bone cementing and bone grafting for giant cell tumour of bone: A systemic review. Ann R Coll Surg Engl. 2019;101(2):79-85. https://doi.org/10.1308/rcsann.2019.0004

\section{PMid:30688529}

8. Coraca-Huber DC, Wurm A, Fille M, Hausdorfer J, Nogler M, Vogt $\mathrm{S}$, et al. Antibiotic-loaded calcium carbonate/calcium sulfate granules as co-adjuvant for bone grafting. J Mater Sci Mater Med. 2015;26(1):5344. https://doi.org/10.1007/ s10856-014-5344-8

PMid:25578698

9. Jia WT, Luo SH, Zhang CQ, Wang JQ. In vitro and in vivo efficacies of teicoplanin-loaded calcium sulfate for treatment of chronic methicillin-resistant Staphylococcus aureus osteomyelitis. Antimicrob Agents Chemother. 2010;54(1):170-6. https://doi.org/10.1128/aac.01122-09

PMid:19917757

10. Kurien T, Pearson RG, Scammell BE. Bone graft substitutes currently available in orthopaedic practice: The evidence for their use. Bone Joint J. 2013;95-B(5):583-97. https://doi. org/10.1302/0301-620x.95b5.30286 PMid:23632666

11. Coraça-Huber DC, Ammann CG, Nogler M, Fille M, Frommelt L, Kühn KD, et al. Lyophilized allogeneic bone tissue as an antibiotic carrier. Cell Tissue Bank. 2016;17(4):629-42. https:// doi.org/10.1007/s10561-016-9582-5

PMid:27631323

12. iDataResearch.USOrthopedicBiomaterials Canada: iData Research Inc.; 2013.

Market-2013.

13. Sayin B, Çaliş $S$, Atılla $B$, Marangoz $S$, Hincal AA. Implantation of vancomycin microspheres in blend with human/rabbit bone grafts to infected bone defects. J Microencapsul. 2008;23(5):55366. https://doi.org/10.1080/02652040600775632

PMid:16980276 